\title{
Relato autobiográfico para la enseñanza de la Naturaleza de la Ciencia: aproximación a la ciencia auténtica a partir de una investigación sobre el dengue
}

\author{
Autobiographic narration for teaching Nature of Science: \\ an approach to authentic science \\ based on an investigation about dengue
}

Fernando Garelli $^{1}$. Silvina Cordero ${ }^{2}$ - Ana Dumrauf ${ }^{2}$

\begin{abstract}
Resumen: Tomando como marco la importancia de abordar las concepciones sobre la Naturaleza de la Ciencia $(\mathrm{NdC})$ y de acercarnos al estudio de una ciencia auténtica (la práctica científica tal y como se desarrolla en laboratorios y otros ámbitos), analizamos la implementación de una secuencia didáctica utilizando el relato autobiográfico de una investigación sobre dengue. El proceso desarrollado puede caracterizarse como de investigación-acción, ya que se efectuó en ciclos sucesivos de planificación, puesta en práctica, análisis y reflexión. La secuencia fue implementada en el nivel universitario y permitió un acercamiento a diversas facetas de la NdC: trayectorias de ingreso al sistema y actores; metodología científica; construcción de hipótesis y desarrollo de diseños experimentales; proceso de escritura de artículos científicos; modas editoriales; e inserción de la ciencia en la trama social y política. La propuesta habría promovido modificaciones en las representaciones de los estudiantes, así como la identificación de sesgos en su formación científica previa.
\end{abstract}

Palabras clave: Naturaleza de la ciencia. Ciencia auténtica. Relatos autobiográficos. Dengue.

\begin{abstract}
Taking as framework the importance of addressing conceptions about Nature of Science (NoS) and approaching the study of authentic science (scientific practice as is actually carried out in laboratories and other areas), we analyze the implementation of a didactic sequence using an autobiographic narration of an investigation about dengue. The process can be characterized as action research, because it was carried out in successive cycles of planning, implementation, analysis and reflection. The sequence was implemented at the university level and allowed approaching various facets of NoS: trajectories of entry into the system and actors; scientific methodology; construction of hypotheses and development of experimental designs; processes of scientific paper writing; editorial fashions; and science as socially and politically embedded. The proposal appears to have promoted changes in the representations of the students and the identification of biases in their previous scientific training.
\end{abstract}

Keywords: Nature of science. Authentic science. Autobiographical chronicles. Dengue.

\footnotetext{
${ }^{1}$ Universidad de Buenos Aires (UBA), Facultad de Ciencias Exactas y Naturales, Buenos Aires, Argentina. E-mail: $<$ fgarelli@gmail.com>

${ }^{2}$ Universidad Nacional de La Plata (UNLP), Facultad de Humanidades y Ciencias de la Educación, La Plata, Buenos Aires, Argentina.
} 


\section{Introducción}

Las concepciones acerca de la Naturaleza de la Ciencia (NdC), y su abordaje en la educación científica, han convocado a numerosos investigadores/as (ABD-EL-KHALICK; BELL; LEDERMAN, 1998; BRICKHOUSE, 1990; HAMMER, 1994). Vázquez Alonso y Manassero Mas (2012) plantean que en la educación en ciencias existen dos tendencias en relación con la demarcación del término NdC: por un lado, aquellos autores que consideran los aspectos epistemológicos, vale decir, rasgos, supuestos y valores inherentes al conocimiento científico; y por otro lado, aquéllos para los que la $\mathrm{NdC}$ engloba aspectos sociológicos e históricos, además de los epistemológicos. Esta última perspectiva atiende especialmente a las relaciones entre ciencia, tecnología y sociedad.

A pesar de las discusiones sobre cuáles serían los contenidos que deberían enseñarse y las formas de hacerlo (ACEVEDO-DÍAZ et al., 2007), algunos autores han intentado definir contenidos enseñables a fin de aportar a la práctica del profesorado (VÁZQUEZ ALONSO; MANASSERO MAS, 2012). Estas definiciones parten de consensos acerca de la NdC tales como los elaborados por Lederman $(1992,2007)$ y su equipo, recuperados por Vázquez Alonso y Manassero Mas (2012). En este sentido, el conocimiento científico se caracterizaría por ser:

[...] provisional (sujeto a cambios); fundamentado empíricamente (basado en y/o derivado de las observaciones del mundo natural); parcialmente subjetivo (cargado de teoría); en parte, producto de la inferencia humana (razonamientos), donde es importante la distinción entre las observaciones e inferencias; en parte también producto de la imaginación y la creatividad (involucra la invención de hipótesis y explicaciones); un conjunto de teorías científicas y leyes, que son entes relacionados y con diferentes funciones; empapado social y culturalmente (VÁZQUEZ ALONSO; MANASSERO MAS, 2012, p. 6).

Algunos autores (IRZIK; NOLA, 2011) han criticado esta mirada de consenso de la $\mathrm{NdC}$ principalmente por ser demasiado restringida, al no reflejar la heterogeneidad existente entre disciplinas científicas, y presentarla como ahistórica e inmutable. Desde el campo de los Estudios de la Ciencia se ha producido un importante cuerpo de conocimientos que plantea una nueva imagen de la ciencia, al centrar la atención en sus procesos de elaboración, desarrollo, negociación y difusión, es decir, la ciencia en elaboración (LATOUR, 1987; LATOUR; WOOLGAR, 1986), en lugar de tomar como objeto de estudio la ciencia ya hecha. En este marco, hemos analizado contextos educativos universitarios caracterizados como ambientes auténticos de aprendizaje (DUMRAUF, 2001). Estos ambientes se conforman como entornos áulicos que comparten características cruciales con los ámbitos de trabajo cotidiano de la comundad científica

Se los ha designado como auténticos para distinguirlos de los ambientes de aprendizaje tradicionales (ROTH, 1997). De acuerdo con Roth (1997), la literatura sobre el trabajo cotidiano de científicos e ingenieros ilustra la naturaleza fundamentalmente social de sus actividades; en tales 
actividades, las personas 'están relacionadas en formas múltiples y heterogéneas' y sus 'ubicaciones sociales, intereses, fundamentos y posibilidades subjetivas son diferentes' (DUMRAUF, 2001, p. 70-71).

Wong y Hodson (2010) resaltaron la importancia del rol que investigadores/as pueden jugar para refinar las visiones respecto a las prácticas de la comunidad científica, la naturaleza de su trabajo, sus objetivos y las relaciones con la sociedad en las que están inmersos. Estos autores reconocen que una forma de promover la comprensión de lo que constituye la ciencia auténtica (la práctica científica como se desarrolla en laboratorios y otros ámbitos) se adquiere participando de los propios ámbitos de investigación (WONG; HODSON, 2010). Sin embargo, este tipo de experiencias no resulta viable para todo el estudiantado, aún en el nivel universitario. Por otro lado, algunos autores acuerdan en que eso no es suficiente y que para desarrollar una mejor comprensión de la $\mathrm{NdC}$ es necesaria la reflexión explícita acerca de estos procesos (BELL et al., 2003; VÁZQUEZ ALONSO; MANASSERO MAS; ORTÍZZ BONNIN, 2013).

El uso de narrativas ha sido planteado como uno de los dispositivos de enseñanza apropiados para trabajar aspectos de la NdC (RIBEIRO; MARTINS, 2007). Faria et al. (2014) demuestran su potencialidad estudiando el uso de relatos imaginados por alumnos/as de $9^{\circ} \mathrm{y}$ $11^{\circ}$ en Portugal. Concluyen que se trató de "un instrumento eficaz para acceder a las ideas de los/as alumnos/as acerca de los científicos y la actividad científica.” (FARIA et al., 2014, p. 17, traducción nuestra). Sin embargo, encuentran limitaciones para dialogar con las ideas estereotipadas de las/os alumnos en sesiones de discusión, en las que tanto docentes como estudiantes se encontraron incómodos. De esta manera, cobra importancia buscar estrategias para aproximarse a la reflexión respecto de la $\mathrm{NdC}$ en el contexto de la ciencia auténtica:

[...] tomando en cuenta estas dificultades, sugerimos que la discusión debe ser integrada en un contexto de trabajo, en el cual los alumnos sean fomentados a establecer ligazones con el trabajo de científicos reales y concretizar los procesos implicados en la construcción de conocimiento científico. (FARIA et al., 2014, p. 18, traducción nuestra)

En este marco hemos elaborado una propuesta didáctica centrada en la utilización del relato autobiográfico de procesos de investigación, como material de enseñanza para posibilitar la reflexión acerca de la $\mathrm{NdC}$ en el aula, y situar al estudiantado en el contexto de la ciencia auténtica. Buscamos promover que pudieran colocarse en el rol de investigadoras/es, a partir de relatos en los que se explicitaron modos de construcción de conocimientos, fundamentaciones, procesos de toma de decisiones, relaciones dentro del grupo de investigación, entre otros aspectos. Esa puesta en situación incluyó la confrontación con preguntas de investigación y la reflexión sobre sus propias representaciones acerca de las prácticas planteadas.

En este trabajo presentamos el análisis y resultados de la implementación de esta propuesta, a partir de un ciclo de investigación-acción llevado a cabo por el primer autor, utilizando como material de enseñanza un relato basado en su experiencia de doctorado sobre la Eco-epidemiología del dengue. 


\section{Metodología}

La actividad analizada en este trabajo fue desarrollada e implementada en el contexto del proyecto "Estudio de representaciones y prácticas en la educación en ciencias naturales, ambiente y salud" (PERSPECNAS), cuyo principal objetivo es analizar prácticas educativas innovadoras y procesos de transformación de representaciones sociales respecto de tópicos específicos del área en diferentes niveles educativos.

El proceso desarrollado puede caracterizarse como de investigación-acción, ya que se implementó en ciclos sucesivos de planificación (a partir de una problemática detectada por el docente-investigador), puesta en práctica con realización de registros y reflexión y revisión de la acción (KEMMIS; MCTAGGART, 2005). Este proceso fue acompañado en las etapas de planificación, análisis y reflexión por investigadoras integrantes del PERSPECNAS, quienes, desde una perspectiva interpretativa, aportaron a la comprensión de los sentidos otorgados por el docente a su acción y al entramado de significaciones construidas en el aula.

El levantamiento de información se realizó a través de audiograbaciones y fotografías de las clases, la realización de un diario docente y la recolección de producciones y evaluaciones de la propuesta por parte del estudiantado. Los registros fueron codificados, categorizados y analizados, siguiendo la perspectiva de análisis de contenido propuesta por Bardin (1977). Una primera fase consistió en un pre-análisis que implicó la realización de lecturas iniciales de los registros a partir de las cuales se delimitaron los momentos de la secuencia didáctica implementada y las dimensiones a analizar en cada uno de ellos. En una segunda fase se puso en práctica lo que el autor denomina “aprovechamiento del material”: operaciones de codificación, descomposición y enumeración en función de consignas formuladas previamente, a partir del marco teórico de referencia y el pre-análisis. La tercera fase fue de "tratamiento de resultados, inferencia e interpretación", a partir de la cual se elaboraron conclusiones en función del trabajo anterior, y su articulación con el marco teórico de referencia.

La secuencia didáctica fue diseñada específicamente para la asignatura Ecología de Poblaciones, perteneciente al ciclo superior de una carrera de Licenciatura en Ciencias Biológicas (Universidad René Favaloro, Buenos Aires, Argentina). Se implementó durante las tercera y cuarta clases del curso, luego de dos primeras clases introductorias a la Ecología de Poblaciones, en las que se trabajó respecto del diseño experimental y las técnicas de muestreo y estimación de abundancia de poblaciones. Cada una de las clases tuvo una duración total de cuatro horas y se realizó en un curso con ocho estudiantes.

\section{Descripción y fundamentación de la secuencia didáctica}

Durante las primeras clases del curso, el alumnado expresó su interés respecto de “dejar de ver contenidos exclusivamente teóricos y estudiar más su aplicación”, ya que se encontraban iniciando su búsqueda de temáticas y orientadores para la realización de un trabajo de tesis con el que finalizarían su carrera de grado. Estas inquietudes, sumadas a las preocupaciones del docente respecto de la importancia de la reflexión acerca de las prácticas investigativas y la $\mathrm{NdC}$, condujeron al diseño de una secuencia didáctica que incluyó un relato de su experiencia reciente como joven investigador en el área de la Eco-epidemiología. 
El relato autobiográfico elaborado se centró en una investigación acerca de la problemática del dengue que se encuentra disponible para ser descargado (GARELLI; CORDERO; DUMRAUF, 2015). Si bien el tema se encuadra en la Biología, el desarrollo de la indagación implicó la participación de diversos actores sociales en diferentes esferas de acción. Buscó describir el proceso de estudio de una estrategia de control del mosquito Aedes aegypti (principal vector del virus de la enfermedad) en la ciudad de Clorinda (Provincia de Formosa, Argentina). Dentro del relato se incluyeron: preguntas de investigación, objetivos, fuentes de información, procesos de elaboración de hipótesis y diseños experimentales, descripción de salidas de campo, procesos de análisis de datos, de escritura y publicación de artículos científicos y propuestas de nuevas estrategias para la superación de la problemática de origen. También contempló aspectos tales como: momento y contexto de formulación del proyecto, marco institucional de la investigación y mecanismos de inserción, conformación de grupos de trabajo y sus relaciones internas, "modas" editoriales y posible impacto social de los resultados de la investigación. Buscó, entonces, ser una narración, desde el punto de vista de uno de los protagonistas, de la ciencia auténtica en un caso particular. Promovería el abordaje de la ciencia como proceso (TOBALDINI et al., 2011), como conocimiento en elaboración, situado social e históricamente.

La secuencia didáctica constó de nueve momentos desarrollados a lo largo de dos clases de 4 horas, separadas por una semana. En el primero, se buscó explicitar representaciones iniciales relacionadas con la $\mathrm{NdC}$ del estudiantado a partir de un cuestionario realizado individualmente y luego discutido por el grupo-clase. Se indagó sobre cómo los estudiantes imaginaban que se ingresa en la investigación, los actores e instituciones con los cuales se vincularían, los pasos en el desarrollo de investigaciones científicas y las actividades que realiza cotidianamente un/a científico/a. Estas representaciones iniciales serían retomadas y discutidas a lo largo de toda la secuencia didáctica.

Luego se realizó una exposición dialogada sobre generalidades de la problemática del dengue, a partir de un torbellino de ideas.

En el tercer momento se inició el trabajo con el relato, cuyo primer fragmento describe la incorporación del joven al grupo de investigación y una primera aproximación al problema objeto de estudio. Se le propuso al estudiantado plantear hipótesis sobre el funcionamiento del sistema bajo estudio en el relato, con el objetivo de identificar posibles limitantes al control del mosquito.

A continuación se trabajó con el segundo fragmento, en el cual se explicitan las hipótesis planteadas por el grupo de investigación del relato. Ello permitió compararlas y ponerlas en diálogo con las construidas por los estudiantes en el momento anterior, analizando sus procesos de elaboración.

En el quinto momento el docente propuso a los y las estudiantes elaborar, en forma individual, un diseño experimental para poner a prueba una de las hipótesis del grupo de investigación del relato. Esto fue enunciado hacia el final de la primera clase y se realizó como tarea para retomar al comienzo de la segunda. Los diseños experimentales construidos fueron puestos en común y comparados con el elaborado por el grupo de investigación (descripto en el tercer fragmento del relato) que fue expuesto por el docente.

En el sexto momento se propuso reflexionar acerca del impacto esperado de la investigación descrita en el relato.

El séptimo momento se basó en el tercer fragmento del relato a partir de la consigna de leerlo individualmente e identificar ejes para discutir en forma plenaria. Los ejes abordados 
en el relato fueron el rol de la escritura de artículos científicos en el proceso de investigación, el rol de los subsidios, el impacto social del trabajo científico, los factores que influyen en la toma de decisiones, la no linealidad de los procesos de investigación.

En el octavo momento se propuso la elaboración de alternativas para futuras investigaciones. Luego, estas propuestas fueron contrastadas con lo planteado por los investigadores del relato mediante la lectura del cuarto fragmento.

Finalmente, se realizó una evaluación de la experiencia y una última reconsideración de las representaciones iniciales; esto se hizo a partir de la redacción de un informe individual en el cual el estudiantado revisó los pasos con los cuales se desarrollan las investigaciones científicas - relevados en el primer momento de la secuencia - y valoraron la propuesta didáctica.

\section{Análisis de los resultados de la implementación}

\section{Momento 1 de la secuencia didáctica: Representaciones iniciales}

A continuación sintetizamos las representaciones identificadas, organizadas según los aspectos relevados.

\section{Ingreso a la investigación}

El estudiantado planteó los siguientes pasos necesarios para insertarse en la investigación como actividad laboral: recibirse (obtener el título de grado), realizar una búsqueda en diferentes grupos de investigación (utilizando como fuentes de información personas conocidas y/o bolsas de trabajo virtuales), comenzar una relación con un grupo de investigación y aplicar a una beca doctoral. Respecto a las becas doctorales, surgieron muchas inquietudes, evidenciando una clara necesidad de conocer mejor los procesos de postulación y las características de las becas disponibles.

La única diferencia entre lo elaborado por las/os estudiantes y lo descrito en el relato autobiográfico fue que, en el relato, el principal actor en la definición de qué hacer a lo largo del doctorado fue el doctorando (algo poco frecuente, pero vivenciado de esa manera por el autor del relato). La comparación entre esta inserción en el grupo de investigación en relación con otros casos, como los que expusieron estudiantes que ya habían comenzando a realizar su tesis de grado, puso de manifiesto la posibilidad de diferentes trayectorias de ingreso para quienes deseen hacer investigación científica.

\section{Actores e instituciones vinculados al proceso de investigación}

En primera instancia, el estudiantado identificó instituciones estatales relacionadas directamente con la investigación, su financiamiento y promoción. Luego señalaron instituciones estatales no directamente relacionadas con la investigación, como parques nacionales u hospitales. Finalmente, mencionaron instituciones privadas, tales como fundaciones financiadoras de proyectos científicos. No se hizo referencia en ningún caso a empresas. En Argentina, la vinculación científica en el área de la Biología con el sector empresarial está poco desarrollada y 
no fue reconocida por el estudiantado presente en el curso. Nuevamente, la trayectoria descrita en el relato, con su particularidad de trabajar cercanamente con una fundación, permitió dar cuenta de la variedad en las posibles interrelaciones institucionales.

Respecto a los actores, se identificaron solamente a "los directores de beca y a los compañeros de trabajo". Sobre la relación entre becarios y directores, el estudiantado expresó que imaginaban la relación "comenzando formal pero luego tendiendo a volverse más informal".

\section{El proceso de investigación}

A partir de las producciones individuales del estudiantado, se construyó en clase un esquema de los pasos a seguir por un/a investigador/a (Figura 1).

Figura 1. Esquema inicial del proceso de investigación científica sistematizado en clase a partir de lo elaborado por el estudiantado

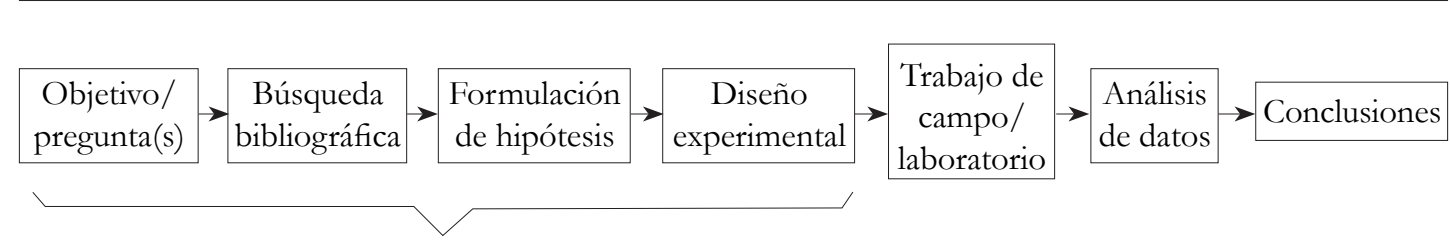

A partir de esto: presentación para subsidios

Fuente: elaborado por los autores.

Este modelo inicial, construido colectivamente, incluyó el seguimiento lineal de una serie de pasos, en forma similar a la enunciación del "método científico" presente aún en algunos libros de texto escolar. Después de la elaboración del modelo, la intervención del docente señaló la homogeneidad de las respuestas, ante lo que uno de los estudiantes explicó que eso ocurría "porque ya lo habian estudiado en otra materia" de esa manera.

La única diferencia con el "método científico" tradicional fue el reconocimiento de la necesidad de solicitar subsidios y la inclusión, a partir de que una de las estudiantes lo sugiriera, de la existencia de trabajo grupal, pero sólo en la etapa relacionada con el diseño experimental.

Se discutió también cómo surgen los proyectos de investigación, sus objetivos y preguntas iniciales y hubo acuerdo entre el estudiantado en que los mismos nacen a partir de la identificación, por parte de expertos/as, de "agujeros" en el conocimiento del área o de una problemática de la "vida cotidiana" o social. De esta manera, desde la perspectiva dicotómica definida por el alumnado, los proyectos surgirían a partir de necesidades teóricas, sin influencias sociales, o bien para contribuir a superar alguna problemática social: "Sabiendo mucho sobre un tema, vos sabés qué agujeros de información hay, o a partir de problemas de la vida cotidiana o, por ejemplo, el medio ambiente, si ves mucha contaminación en un lugar, te surge, ¿cómo podría remediarlo o evitar que empeore?’ 


\section{Momentos 3, 4 y 5 de la secuencia didáctica: desarrollo de hipótesis y diseños experimentales}

\section{Construcción de hipótesis}

El momento de plantear hipótesis es un momento creativo que juega un papel fundamental en todas las etapas de una investigación, como puede inferirse del relato en el que varias veces se vuelve sobre diferentes hipótesis elaboradas, en contraste con lo que suele encontrarse en libros de texto. (WONG; HODSON, 2009).

Las hipótesis planteadas por el estudiantado respecto a las posibles fallas al control del mosquito, fueron similares a las de los investigadores del relato, excepto que el alumnado no identificó una de ellas (que el programa de control no reconociera ciertos tipos de recipientes como posibles criaderos del mosquito).

En este punto resulta interesante resaltar algunos aspectos del proceso de construcción de hipótesis que aparecen en el relato. En primer lugar, el joven científico nos aclara que para revisar los pasos de la estrategia de control resultó "fundamental conversar tanto con operarios de la Fundación como con dueños de casas" (GARELLI; CORDERO; DUMRAUF, 2015, p. 4). Si bien las preguntas a operarios estuvieron pautadas, las conversaciones con dueños de casa no estaban previstas ni preestablecidas. En este sentido, se constituyeron, sin preverlo, en una fuente relevante para la construcción de nuevas hipótesis. En estas hipótesis se reflejan también algunas componentes sustentadas por la observación y otras por el bagaje teórico del investigador. Si bien la observación posee siempre bases teóricas, diferenciamos para el análisis de estas hipótesis las vertientes observacional, no intencional y teórica, reconociendo que todas poseen teorías subyacentes.

De esta manera, el relato expone diferencias respecto del surgimiento de las hipótesis con lo elaborado inicialmente por los/as estudiantes. En principio, al estudiantado le costó reconocer esto como algo verdaderamente diferente a lo que habían elaborado, no encontraban diferencia entre formular una hipótesis a partir de lo leído en la bibliografía o a partir de hablar con un poblador. Sin embargo, a través de las reflexiones realizadas en clase, incluyeron la posibilidad de que otras fuentes de información influyeran en el proceso: "Claro, fue diferente, a partir del trabajo de campo, de hablar con la gente, se planteó una nueva hipótesis, incluso a partir de las conclusiones a las que uno va llegando pueden surgir nuevas hipótesis".

\section{Diseño experimental}

La primera clase se cerró con el pedido de elaboración extra-clase de una propuesta de diseño experimental para poner a prueba una de las hipótesis del relato: "Que el efecto del temefós [larvicida utilizado en el control del dengue] no durara tanto como se esperaba." (GARELLI; CORDERO; DUMRAUF, 2015, p. 4).

Los diseños experimentales propuestos por los/as estudiantes fueron diferentes de los del grupo de investigación del relato, dado que todos se pensaron en condiciones de laboratorio, mientras que lo descripto en el relato incluyó actividades en campo. Todos sus diseños plantearon la realización de bioensayos semanales de mortalidad de larvas en recipientes tratados 
con temefós en el laboratorio. En algunos casos, proponían además simular el recambio de agua en los tanques.

En el relato, el grupo de investigación realizó bioensayos como los planteados por los/ as estudiantes, pero tomando muestras de agua de tanques tratados en las casas de Clorinda, es decir en condiciones de campo, que incluyen las prácticas de recambio y manejo de agua de los habitantes del lugar. En este sentido, los diseños elaborados por los/as estudiantes no fueron adecuados para poner a prueba la hipótesis en las condiciones del sitio de estudio.

Ninguno de los estudiantes pudo derribar las paredes del laboratorio. Al reflexionar colectivamente sobre por qué ocurrió esto, se llegó a la conclusión de que "nunca en otras materias se habia tenido que pensar diseños para poner a prueba hipótesis fuera del laboratorio". De esta manera, se evidenció un sesgo en su formación previa.

\section{Momentos 6 y 7 de la secuencia didáctica: las publicaciones científicas y su impacto social}

A partir de la lectura del tercer fragmento del relato surgieron múltiples y agitadas discusiones respecto del proceso científico y de lo descripto allí con relación a la NdC. Los tópicos discutidos incluyeron algunos de los esperados a priori (la escritura de artículos científicos, la influencia de modas editoriales o intelectuales, el impacto social de la investigación) y otros no desarrollados en profundidad en el relato, tales como la responsabilidad del científico, los conflictos de intereses y el entramado político-social en el que se inserta la investigación.

\section{La escritura de artículos científicos}

Los procesos de validación y aceptación de los hallazgos científicos son aspectos cruciales de la dimensión social de la práctica científica. Los resultados, conclusiones y nuevas hipótesis formuladas por investigadores/as, deben ser presentados, discutidos y argumentados de acuerdo con formas y lenguajes determinados por la comunidad científica. "La confianza de un científico en el significado de su trabajo es insuficiente para establecerlo como parte del conjunto de conocimientos, debe resistir el escrutinio crítico de la comunidad por lo que significa que la comunidad decide si es apropiado." (WONG; HODSON, 2010, p. 21, traducción nuestra).

De acuerdo con el relato, al joven científico le llevó mucho tiempo elaborar sus artículos porque necesitó transitar un proceso de aprendizaje de códigos y estilos usuales en la literatura del área, y requirió intercambios con su director (conocedor de dichos códigos y estilos). Se estableció entre ellos un proceso que involucró ciclos de escritura del joven investigador y correcciones del director, ajustados a los escasos tiempos disponibles de este último. Este proceso llamó la atención del estudiantado, dado que desconocían los procedimientos para elaborar publicaciones y no habían incluido esta actividad como parte del proceso de investigación inicialmente planteado por ellos. A su vez, resultó sorprendente para los/as estudiantes que uno de los trabajos se hubiera publicado con dificultad y luego de algunas correcciones solicitadas por los revisores: "No sabia que existian tantas idas y vueltas a la hora de escribir un paper pero más me llama la atención que les haya costado tanto publicarlo, dado que pareciera que es un trabajo relevante." 


\section{La influencia de modas editoriales o intelectuales}

El joven investigador del relato explicita la influencia de sus lecturas para la elaboración de las preguntas de investigación. Considera "una especie de moda" las estrategias de relevamiento, que fueron cambiando con el tiempo. Atendiendo a que estos cambios de estrategias fueron relevadas en las publicaciones, el joven científico estaría dando cuenta de lo que Wong y Hodson (2010) denominan "moda intelectual", descrita por diferentes integrantes de la comunidad científica como la orientación de la política editorial de las publicaciones periódicas. $\mathrm{Al}$ estudiantado no le resultó sorprendente esta idea, de acuerdo con uno de ellos, esto no era novedoso dado que el trabajo científico "es un trabajo bumano como cualquier otro $y$, por lo tanto sujeto a que existan modas".

\section{Impacto social de la investigación, responsabilidad del/a científico/a, conflictos de intereses y entramado político-social}

Lo que más movilizó a los y las estudiantes fue la falta de impacto social del trabajo relatado. Los/as estudiantes previamente habían supuesto que la investigación tendría un fuerte impacto y que generaría un cambio en las estrategias de control del mosquito por parte de las dependencias político-sanitarias pertinentes. El bajo impacto por fuera de la esfera de la comunidad científica generó diversas discusiones, buscando entender las razones. Las reflexiones derivaron hacia la responsabilidad de los científicos, los posibles conflictos entre el trabajo científico e intereses económicos y la interrelación entre ciencia, sociedad y modelo político-económico. Estos tópicos no están explícitamente tratados en el relato pero aparecieron a partir de la problemática planteada.

En el relato, el trabajo del joven investigador finalizó con la publicación de sus resultados. Eso fue criticado por los/as estudiantes, quienes argumentaron que los/as científicos/ as deberían también actuar más allá de la esfera académica:

Estudiante 1: A mi me parece que, como me dijo una amiga hace mucho tiempo: 'Vos esperás mucho de los demás. Tenés que esperar menos y hacer más'. Entonces, hay que tomar en cuenta eso. Vos estás esperando mucho del ministerio de salud. [...]

Estudiante 2: Pero qué, vos vas al ministerio de salud y les decís que 'esto no funciona, hacé algo para solucionarlo' y listo, ¿̇ya está? ¿'Te vas a tu casa? [...]

Estudiante 1: Pero vos vas y hacés tu investigación ¿y qué? Y después hacés otra ¿y qué? ¿dejás todo abi tirado? [...]

Estudiante 3: Hay muchos científicos cómodos que no les interesa investigar en pos de la bumanidad, sólo investigan en pos de lo que ellos quieren.

Estos cuestionamientos llevaron a considerar al trabajo del científico dentro del entramado político-social y como parte de posibles conflictos de intereses. La investigación relatada dio cuenta de que el insecticida en condiciones de campo no resultaba tan adecuado como se 
esperaba. Los/as estudiantes infirieron que este hallazgo podría traer conflictos con quienes organizan las campañas de control y con quienes producen el insecticida:

Estudiante 3: No es ese el problema. El problema es que no lo quieren erradicar, es que no se persigue el bien de la sociedad, se persigue el ganar plata.

Estudiante 4: Se financian investigaciones mientras sirvan a sus intereses y economía.

$[\ldots]$

Estudiante 5: Para mí bubo problemas políticos.

Estudiante 1: O sea, está bueno que vos digas 'Yo voy a investigar cuánto dura el temefós', ahora... no me conviene que se deje de usar. Entonces viene por ese lado.

Estudiante 3: Para mí pasa por ahi, por decir mirá, estamos controlando el dengue... si después funciona o no... para mí al que está bancando la campaña ... ¿qué le importa si anda o no?

\section{Momento 8 de la secuencia didáctica: alternativas a seguir en el proceso de investigación}

La pregunta acerca de cómo transformar lo existente lleva a diferentes alternativas de acción, más allá de la comprensión o explicación acerca del funcionamiento de la estrategia de control. Como se describe en el relato, el joven científico, posiblemente con base en sus convicciones personales acerca de para qué sirve la ciencia, no se limita a continuar sólo en el camino de los resultados "biológicos", sino que se plantea alternativas que impacten sobre la realidad social local, tanto a través de la participación ciudadana, como de la implementación de políticas públicas que garanticen el derecho al agua y a la salud. Sus propios ideales lo llevan a ampliar sus opciones.

Los valores personales, las prioridades y las experiencias anteriores dictan hacia dónde y cómo los científicos dirigen su trabajo. Además, la ciencia es un empeño humano y está influenciada por la sociedad y la cultura donde se desarrolla. Los valores de la cultura determinan el rumbo de la ciencia, cómo lo persigue, se interpreta, se acepta y se utiliza (VÁZQUEZ ALONSO; MANASSERO MAS, 2012, p. 7).

En diversos momentos de la clase surgieron opiniones sobre qué hacer respecto de la problemática y en consonancia con lo descripto, el estudiantado apuntó a un abordaje más integral que incluyera la educación y el trabajo comunitario en primera instancia. El relato los habría estimulado a buscar soluciones procedentes de otros campos. Luego de ser interrogados sobre otras posibles alternativas, produjeron las mismas que se describen en el relato, excepto el proporcionarles agua de red de buena calidad a los habitantes de Clorinda: "hacer algo para que la comunidad participe desde la educación y la comunicación, buscar mejores larvicidas, ponerles tapas a los recipientes de la gente", e "investigar cómo hacer un control biológico, por ejemplo con peces". 


\section{Momento 9 de la secuencia didáctica: cambios en las representaciones sobre el proceso de investigación y evaluación de la propuesta didáctica}

Luego de las clases, los esquemas elaborados sobre el proceso científico fueron modificados por todos/as los/as estudiantes. Alteraron el carácter lineal del proceso, incluyendo múltiples ciclos de realimentación (véase un ejemplo en la Figura 2). Las etapas no fueron modificadas, con excepción de la inclusión de la escritura de artículos científicos en algunos casos.

Figura 2. Esquema del proceso de investigación científica elaborado por una estudiante luego de la implementación de la secuencia didáctica

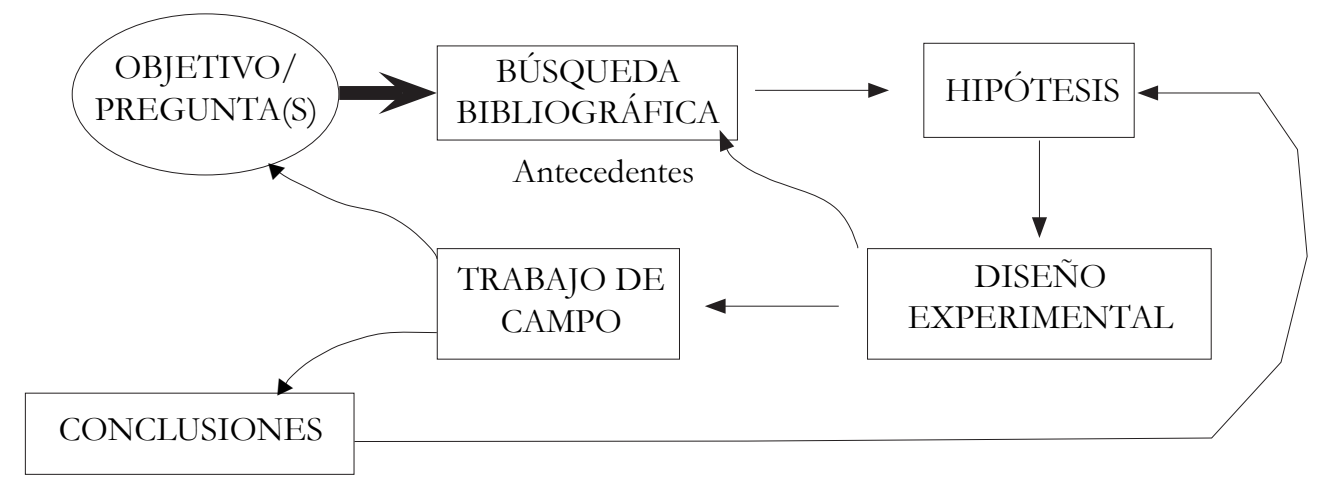

Fuente: Producciones de estudiantes post-secuencia.

Estas modificaciones en los esquemas evidenciarían cambios en las representaciones del estudiantado, fundamentalmente en relación con la linealidad del proceso.

La secuencia didáctica implementada fue evaluada positivamente por todos/as los/as estudiantes, quienes valoraron su carácter dialógico, la aproximación que les ofreció al proceso de trabajo científico y sus implicancias sociales, vale decir el acercamiento a la ciencia auténtica y la $\mathrm{NdC}$ propuesto. En general las valoraciones fueron completamente positivas, exceptuando un comentario del segundo extracto que presentamos a continuación:

Me pareció muy buena la propuesta, no sólo entretenida sino también práctica y dinámica. Aprendi mucho, y no sólo de teoría de muestreo o cómo realizar un trabajo experimental, sino también sobre lo que hace un biólogo cientifico, cómo es la sociedad en la que vamos a trabajar, y cosas a tener en cuenta en el futuro que nos van a ayudar a desenvolvernos como profesionales. 
La propuesta de la clase está buena sobre todo porque es distinto a lo que hacemos en todas las otras materias, es más interactiva y nos da una idea más certera de lo que va a ser nuestro trabajo cuando dejemos la universidad. Nos hace pensar en las cosas inesperadas que pueden pasarnos, que capaz todavía no nos lo habiamos planteado seriamente. Como cosas nuevas me llevo una idea más clara de lo que va a ser el doctorado o el trabajo como investigadora porque nunca nos explicaron los pasos a seguir, cómo haces para conseguir plata, un grupo de estudio [...], teníamos suposiciones obviamente, pero abora está más claro. Capaz, haya que ajustar algún detalle de los ejercicios de la clase, pero en general está bueno tener clases así.

Con respecto a las ideas nuevas que nos llevamos, en mi caso fue la de cómo se lleva a cabo una investigación, que no siempre tenemos que bacer un modelo tan estricto como el que habiamos propuesto. Que la búsqueda bibliográfica no es sólo la de los libros, sino que se pueden hacer salidas de campo y analizar los datos obtenidos y a partir de ellos plantear una nueva pregunta.

\section{Discusión}

La secuencia implementada permitió un acercamiento a diversas aristas del poliédrico conocimiento de la NdC (ACEVEDO-DÍAZ et al., 2007), en el contexto de la ciencia auténtica. Las facetas trabajadas pueden resumirse en: diferentes trayectorias de ingreso al sistema y actores con quienes se interrelacionan los/as científicos/as; metodología científica; construcción de hipótesis y desarrollo de diseños experimentales; proceso de escritura de artículos científicos; modas editoriales; y múltiples aspectos relacionados con la ciencia inserta en una trama social y política. La implementación habría fomentado modificaciones en las representaciones de las/ os estudiantes al respecto, así como la identificación de sesgos en su formación.

Las facetas mencionadas pueden relacionarse con los cuatro aspectos sociales de la ciencia que merecen atención en el curriculum escolar según Weinstein (2008):

[...] la ciencia como investigación (es decir, la ciencia como un conjunto de prácticas acordadas); la ciencia como trabajo (reconociendo que la ciencia no es sólo cuestión de conducir experimentos para probar nuevas hipótesis; implica solicitar subsidios, conferencias, críticas, y publicaciones); la ciencia como empresa (tratando con beneficios, patentes, economía política, interés, lobbys, legislaciones y financiamiento) y la ciencia como cultura (ciencia como embebida en contextos culturales, políticos e históricos más amplios) (WEINSTEIN, 2008 apud WONG; HODSON, 2010, p. 4).

Respecto a la ciencia como investigación, los/as estudiantes expresaron cambios en sus representaciones metodológicas a partir del trabajo realizado, complejizando su modelo de "método científico" que comenzó siendo lineal para incluir retroalimentaciones, así como nuevos pasos en algunos casos. Una cuestión que merecería ser complejizada en futuras implementaciones sería los diversos procesos que originan proyectos de investigación. A su vez, 
los/as estudiantes consideraron que lo realizado les permitió tener una "idea más certera de lo que va a ser nuestro trabajo". En ese sentido, en sus valoraciones finales expresaron que la secuencia didáctica posibilitó un acercamiento más fiel al trabajo científico, es decir, a la ciencia auténtica. Estos cambios probablemente estén relacionados con la naturaleza del relato, que describe los pasos y prácticas realizados tal como fueron vivenciados por uno de sus protagonistas en una investigación real.

Los interrogantes surgidos relacionados con la ciencia como trabajo, involucraron, fundamentalmente, demandas de información. Tanto los procesos de solicitud de becas como los relacionados a la escritura y publicación de artículos permitieron al estudiantado informarse sobre prácticas que desconocían.

Los aspectos de la ciencia como empresa y como cultura fueron tratados en forma entrelazada y en relación con el impacto social de la investigación. Se suscitaron discusiones no explícitamente planteadas por la secuencia, relacionadas con las dimensiones ético-políticas de la ciencia, tales como la responsabilidad de las y los científicos y los posibles conflictos de intereses entre distintos actores sociales frente a los resultados de una investigación. Los/as estudiantes expresaron posturas que demandaron mayor compromiso social de los/as científicos/as, alejándose del modelo de neutralidad valorativa. Esta concepción hegemónica indica que la actividad científica no debe responder por cómo se utilizan sus productos (HELER, 1996). Por el contrario, las opiniones de los/as estudiantes apuntaron a un mayor compromiso social respecto de las problemáticas relacionadas con la investigación. En estos aspectos, se evidenció una aparente contradicción en los/as estudiantes quienes aceptaron, sin conflicto, la existencia de "modas editoriales" pero se manifestaron sorprendidos por el bajo impacto social de los resultados obtenidos en la investigación. Aún percibiendo la ciencia como emprendimiento social y alejándose de un modelo de neutralidad valorativa, estarían colocándola en un posicionamiento altruista, desconociendo parte de las tensiones y contradicciones propias de las relaciones sociales.

Por otro lado, la secuencia didáctica permitió abordar procesos clásicamente considerados científicos, como el diseño experimental y el planteamiento de hipótesis. De esta manera, se pusieron en juego contenidos propios de la asignatura en la que se implementó la secuencia (Ecología de Poblaciones), tales como técnicas de muestreo y estimación de la abundancia. Estas prácticas posibilitaron poner en cuestión ideas estereotipadas de las/os estudiantes (la actividad experimental sólo se realiza en laboratorios). En este sentido, la vinculación con prácticas de investigación concretas habría permitido la problematización de estas ideas y la identificación de sesgos en la formación previa.

Teniendo en cuenta los aspectos mencionados, la secuencia didáctica implementada habría cumplido el objetivo de aproximarse a la $\mathrm{NdC}$ en el marco de la ciencia auténtica.

\section{Reflexiones finales}

Frente a las dificultades para la participación del estudiantado en procesos de investigación científica reales, especialmente en algunos contextos, la posibilidad de recrear la práctica mediante casos de estudio aparece como una alternativa potente (WONG et al., 2008). La utilización de una narración de un proceso de investigación como material de enseñanza 
puede tener varias ventajas, como la relativa facilidad de construcción y la gran versatilidad que presenta, al poder enfocarse en diversos aspectos de la historia. Asimismo, la contemporaneidad del relato y su redacción en primera persona permitirían a los/as estudiantes empatizar más con el/la investigador/a, y apropiarse simbólicamente de su rol para reflexionar sobre el tema.

En la secuencia didáctica implementada, estas características parecen haberse expresado, contribuyendo a lograr los objetivos propuestos. Sin embargo, sólo puede considerarse una aproximación inicial a la NdC. Un abordaje más amplio y profundo, debería incluir esta perspectiva en diversas instancias de formación, permeando diferentes asignaturas científicas.

El uso de relatos autobiográficos en secuencias didácticas como la analizada en este trabajo resultaría una estrategia versátil, adaptable a otros contextos, niveles educativos y asignaturas. Para ello, es necesario atender a los nuevos desafíos que implicaría para la formación docente y a las condiciones para el desarrollo de otros relatos y secuencias didácticas.

En niveles pre-universitarios, los y las docentes no suelen tener experiencia en el desarrollo de procesos de investigación. Por ello, la utilización de este tipo de secuencias, así como toda aproximación a la ciencia auténtica, requerirían la implementación de procesos de formación docente que apunten a profundizar y complejizar sus representaciones, así como a ampliar los repertorios de intervención.

En cuanto a la construcción de nuevos relatos autobiográficos, sería necesario realizar entrevistas a científicos/as que permitan reconstruir sus investigaciones, buscando específicamente indagar en las aristas particulares de la $\mathrm{NdC}$ en cada caso.

En este sentido, los relatos constituirían una forma de presentación de investigaciones desde la perspectiva de la ciencia auténtica, alternativa a los artículos científicos ortodoxos, cuya estructura, como indicó Medawar (1963, p. 1, traducción nuestra), "representa equívocamente el proceso de pensamiento que acompañó o generó el trabajo". De todas formas, los relatos sólo deben considerarse como una aproximación a ese proceso, una fuente primaria que expresa el punto de vista de su autor y de ninguna manera el "verdadero" proceso de investigación.

Consideramos que esta propuesta constituye un aporte a la formación de estudiantes universitarios/as de carreras científicas, pues permite evidenciar actividades y decisiones propias de la labor científica, habitualmente no abordadas en las asignaturas de grado. Contribuye asimismo a forjar un perfil de científicos/as críticos/as, reflexivos/as sobre su propia actividad y comunidad de pertenencia. Además, en este relato en particular, se apunta a valorar la ciencia desarrollada con compromiso social.

\section{Referencias}

ABD-EL-KHALICK, F.; BELL, R. L.; LEDERMAN, N. G. The nature of science and instructional practice: making the unnatural natural. Science Education, Hoboken, v. 82, n. 4, p. 417-436, 1998.

ACEVEDO-DÍAZ, J. A. et al. Consensos sobre la naturaleza de la ciencia: fundamentos de una investigación empírica. Revista Eureka sobre Enseñanza y Divulgación de las Ciencias, Cadiz, v. 4, n. 1, p. 42-66, 2007. Disponible en: <http://www.redalyc.org/articulo. oa?id=92040104 >. Acceso el: 25 nov. 2015. 
BARDIN, L. Análisis de contenido. 2. ed. Madrid: Akal, 1977.

BELL, R. L. et al. Just do it?: impact of a science apprenticeship program on high school students' understandings of the nature of science and scientific inquiry. Journal of Research in Science Teaching, Hoboken, v. 40, n. 5, p. 487-509, 2003.

BRICKHOUSE, N. W. Teachers' beliefs about the nature of science and their relationship to classroom practice. Journal of Teacher Education, Thousand Oaks, v. 41, n. 3, p. 53-62, 1990.

DUMRAUF, A. "Esas otras cosas que se enseñan que no son física": imágenes de ciencia y prácticas docentes en una experiencia universitaria de enseñanza de física. Investigações em Ensino de Ciências, Porto Alegre, v. 6, n. 1, p. 57-78, 2001. Disponible en: <http://www. if.ufrgs.br/ienci/artigos/Artigo_ID69/v6_n1_a2001.pdf >. Acceso el: 25 nov. 2015.

FARIA, C. et al. “Como trabalham os cientistas?”: potencialidades de uma atividade de escrita para a discussão acerca da natureza da ciência nas aulas de ciências. Ciência \& Educação, Bauru, v. 20, n. 1, p. 1-22, 2014. Disponible en: <http://dx.doi. org/10.1590/1516-731320140010002 >. Acceso el: 24 nov. 2015.

GARELLI, F.; CORDERO, S.; DUMRAUF, A. Reflexiones en el aula sobre la naturaleza de la ciencia: un relato autobiográfico y una secuencia didáctica en base a una investigación sobre el dengue. La Plata: Universidade Nacional de La Plata, 2015. Disponible en: < http:// goo.gl/hNxddk>. Acceso el: 24 nov. 2015.

HAMMER, D. Epistemological beliefs in introductory physics. Cognition and Instruction, London, v. 12, n. 2, p. 151-183, 1994.

HELER, M. Ética y ciencia: la responsabilidad del martillo. Buenos Aires: Biblos, 1996.

IRZIK, G.; NOLA, R. A family resemblance approach to the nature of science for science education. Science \& Education, Dordrecht, v. 20, n. 7, p. 591-607, 2011.

KEMMIS, S.; MCTAGGART, R. Participatory action research: communicative action and the public sphere. In: DENZIN, N. K.; LINCOLN, Y. S. (Ed.). The Sage handbook of qualitative research. 3rd. ed. London: Sage, 2005. p. 559-603.

LATOUR, B. Science in action. Cambridge: Harvard University Press, 1987.

LATOUR, B.; WOOLGAR, S. Laboratory life: the construction of scientific facts.

Princeton: Princeton University Press, 1986.

LEDERMAN, N. G. Nature of science: past, present, and future. In: ABELL, S. K.;

LEDERMAN, N. G. (Ed.). Handbook of research on science education. Mahwah:

Lawrence Erlbaum, 2007. p. 831-880.

. Students' and teachers' conceptions of the nature of science: a review of the

research. Journal of Research in Science Teaching, Hoboken, v. 29, n. 4, p, 331-359, 1992.

MEDAWAR, P. B. Is the scientific paper a fraud? The Listener, Auckland, v. 70, p. 377-378, 1963. 
RIBEIRO, R. M. L.; MARTINS, I. O potencial das narrativas como recurso para o ensino de ciências: uma análise em livros didáticos de física. Ciência \& Educação, Bauru, v. 13, n. 3, p. 293-309, 2007. Disponible en: <http://dx.doi.org/10.1590/S1516-73132007000300002>. Acceso el: 25 nov. 2015.

TOBALDINI, B. G. et al. Aspectos sobre a natureza da ciência apresentados por alunos e professores de licenciatura em ciências biológicas. Revista Electrónica de Enseñanza de las Ciencias, Vigo, v. 10, n. 3, p. 457-480, 2011. Disponible en: <http://www.saum.uvigo. es/reec/volumenes/volumen10/REEC_10_3_4.pdf $>$. Acceso el: 25 nov. 2015.

VÁZQUEZ ALONSO, A.; MANASSERO MAS, M. A. La selección de contenidos para enseñar naturaleza de la ciencia y tecnología (parte 1): una revisión de las aportaciones de la investigación didáctica. Revista Eureka sobre Enseñanza y Divulgación de las Ciencias, Cadiz, v. 9, n. 1, p. 2-31, 2012.

VÁZQUEZ ALONSO, A.; MANASSERO MAS, M. A.; ORTÍZ BONNIN, S. Análisis de materiales para la enseñanza de la naturaleza del conocimiento científico y tecnológico. Revista Electrónica de Enseñanza de las Ciencias, Vigo, v. 12, n. 2, p. 243-268, 2013. Disponible en: <http://reec.uvigo.es/volumenes/volumen12/reec_12_2_2_ex691.pdf>. Acceso el: 25 nov. 2015.

WONG, S. L.; HODSON, D. From the horse's mouth: what scientists say about scientific investigation and scientific knowledge. Science Education, Hoboken, v. 93, n. 1, p. 109$130,2009$.

More from the horse's mouth: what scientists say about science as a social practice. International Journal of Science Education, Abingdon, v. 32, n. 11, p. 1431-1463, 2010.

WONG, S. L. et al. Turning crisis into opportunity: enhancing student-teachers' understanding of nature of science and scientific inquiry through a case study of the scientific research in severe acute respiratory syndrome. International Journal of Science Education, Abingdon, v. 30, n. 11, p. 1417-1439, 2008. 